\title{
Mullerian adenosarcoma (heterologous) of the cervix with sarcomatous overgrowth: a case report with review of literature
}

\author{
Rajan Duggal ${ }^{1}$, Raje Nijhawan ${ }^{1}$, Neelam Aggarwal ${ }^{2}$, Pooja Sikka ${ }^{2}$ \\ Departments of ${ }^{1}$ Cytopathology and Gynecologic Pathology, ${ }^{2}$ Obstetrics and Gynecology, \\ Postgraduate Institute of Medical Education and Research, Chandigarh, India
}

\begin{abstract}
Mullerian adenosarcoma is a rare biphasic malignant neoplasm of the cervix characterized by an admixture of benign epithelial elements and a malignant sarcomatous stromal component, which may be either homologous or heterologous. An aggressive variant of adenosarcoma, mullerian adenosarcoma with sarcomatous overgrowth (MASO) is extremely rare, with only two such cases being reported in the English literature to date. In this report we present a case of MASO of uterine cervix with heterologous elements in a 15-year-old unmarried girl presenting with foul smelling menstrual bleeding and passage of fleshy masses. Because MASO with heterologous elements seems to appear at the earliest stages of reproductive lifespan in women, and have an uncertain malignant potential, gynecologists and pathologists should be aware and think about the possibility of this tumor.
\end{abstract}

Key Words: Mullerian adenosarcoma, Sarcomatous overgrowth, Heterologous, Uterine cervix

\section{INTRODUCTION}

The term mullerian adenosarcoma (MA) was introduced in 1974 to designate an uncommon variant of mullerian mixed tumor of the uterus characterized by a benign, but occasionally atypical glandular component and a sarcomatous stromal component, which is usually low-grade. ${ }^{1}$ In contrast to typical malignant mullerian mixed tumors (MMMTs), MA are usually tumors of low malignant potential. It is a rare neoplasm typically arising in the endometrium, but has also been reported to occur in the ovary, cervix and extrapelvic sites as well. ${ }^{2}$ Mullerian adenosarcoma with sarcomatous overgrowth (MASO) is a rare variant of uterine adenosarcoma, associated with aggressive clinical course, postoperative recurrence, metastasis and a fatal outcome. ${ }^{3}$ The diagnosis of MASO is considered when a pure sarcoma, similar to, or of higher grade than that of underlying adenosarcoma, accounts for at least one-quarter of the tumor after thorough histological sampling of the hysterectomy specimen. MASO in the cervix is an extremely rare entity with only two cases being reported to date

Received July 13, 2009, Revised September 26, 2009,

Accepted October 5, 2009

\section{Correspondence to Rajan Duggal}

Department of Cytopathology and Gynecologic Pathology, Postgraduate Institute of Medical Education and Research, Chandigarh 160012, India

Tel: 91-172-2755117, Fax: 91-172-2744401

E-mail: duggal.rajan@gmail.com in the English literature, which occurred in a middle-aged and an elderly female and clinically presented like a cervical polyp, however in none of these cases were heterologous elements identified. ${ }^{4,5}$ In this report, we describe a case of MASO of the uterine cervix with heterologous elements which occurred in a young unmarried girl. To the best of our knowledge such a case has not been reported in the English literature before.

\section{CASE REPORT}

A 15-year-old unmarried girl, previously healthy, presented with irregular, heavy, foul smelling menstrual bleeding with passage of fleshy masses for two months. She was investigated for menorrhagia and the ultrasound abdomen was suggestive of a fibroid of the uterus. With these complaints she was referred to our institute. Here on abdominal examination the uterus was 18 weeks size and pelvic examination revealed a fleshy necrotic mass protruding through the introitus and occupying the whole vagina. Repeat ultrasonography during this time showed a solid-cystic mass in the anterior wall of the uterus suggestive of degenerated fibroid, and another heterogeneous mass protruding through the vagina which was suggested as polyp. Magnetic resonance imaging (MRI) showed a diffusely enlarged uterus measuring $21 \times 11 \times 11 \mathrm{~cm}$ with evidence of multiple ill defined nodules which were blending with each other and replacing the uterine parenchyma completely. The largest nodule measured $7 \times 6 \times 6 \mathrm{~cm}$. There was no evidence of free fluid or pelvic lymphadenopathy. MRI features were suggestive of diffuse leiomyoma- 
tosis, however a rare possibility of an aggressive lesion was also entertained. Hematological investigations revealed a low hemoglobin level of $7.5 \mathrm{mg} / \mathrm{dL}$ and normal platelet count. Total leukocyte count was $16.0 \times 10^{9} / \mathrm{L}$ with a neutrophilic predominance. Erythrocyte sedimentation rate was normal. Coagulation studies were within normal limits. Biochemical work up revealed a blood urea $24.0 \mathrm{mg} / \mathrm{dL}$ and serum creatinine $0.5 \mathrm{mg} / \mathrm{dl}$. Routine urine examination and culture was negative. Chest X-ray was normal. The vaginal mass was removed piecemeal and sent for histopathology examination with culture and sensitivity with clinical impression of infected fibroid sarcoma. The paraffin embedded section showed predominantly putrefactive necrosis and a tumor composed of interlacing fascicles of elongated atypical spindle cells with high mitotic activity. In addition, heterologous elements in the form of lobules of immature cartilage and rhabdomyoblasts were also noted. Based on these histological findings the possibility of sarcoma with heterologous elements was suggested. In view of the clinical and histopathological features suggestive of a sarcoma, possibly of cervical origin, total abdominal hysterectomy with bilateral salpingo-oophorectomy and omentectomy was done. In addition peritoneal washings were sent for cytological examination.

Intra-operatively, the uterine fundus and body appeared mildly bulky and pushed up by the distended vagina. The cervix was replaced by a friable necrotic tumor. No normal cervical tissue was identified. The bladder wall was edematous and drawn up by the distended vagina. In addition a $1 \times 1 \mathrm{~cm}$ nodule present in the posterior parietal peritoneum was also sampled to exclude a metastatic deposit. Postoperative course was uneventful. The patient was discharged in a satisfactory condition.

\section{Pathologic findings}

The specimen of total abdominal hysterectomy with bilateral salpingo-oophorectomy was received for histopathological examination. On gross examination, friable congested growth was seen arising from the lower uterine segment and involving whole of cervix. Rest of the tumor was received piecemeal with the largest tumor fragment measuring $6 \times 5$ $\mathrm{cm}$. The cut surface of the tumor was fish-flesh or gelatinous, and varied from pinkish-white to gray tan to yellow. The adjoining myometrium and endometrial cavity were grossly free of tumor. The bilateral adnexae were unremarkable. The separately sent metastatic tumor deposit had a similar gross appearance. The omentectomy specimen revealed no gross tumor deposits.

On microscopic examination, the tumor was characterized by intimate admixture of bland endocervical glandular component and sarcomatous stromal component. The endocervical glands were surrounded by a sarcomatous stroma that typically exhibited periglandular condensation and focally polypoidal projection into the glands (Fig. 1). The stromal component focally resembled a low grade stromal sarcoma (consti-

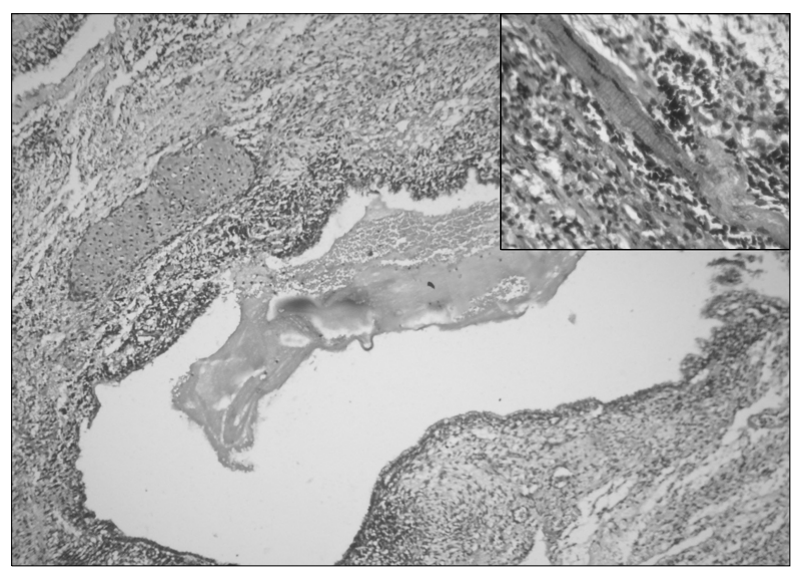

Fig. 1. Endocervical glands surrounded by a sarcomatous stroma that typically exhibits periglandular condensation and focally polypoidal projection into the glands.

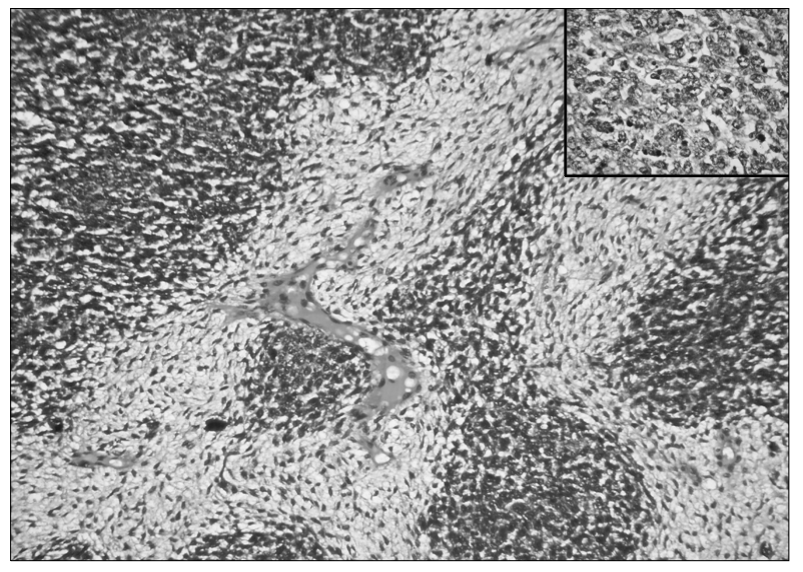

Fig. 2. Higher power view of the malignant stroma, demonstrating a clear demarcation between the low grade and high grade stromal components.

tuting $30 \%$ tumor area), however, the majority was composed of high grade poorly differentiated sarcoma (constituting $70 \%$ tumor area). In the low grade sarcomatous areas the mitotic rate was low (3 mitotic figures [MFs]/10 high power fields [HPFs]), however the mitotic rate was high (24 MFs/10 HPFs) in the poorly differentiated sarcomatous areas. A clear demarcation was noted between the low grade and high grade stromal components (Fig. 2). In addition, heterologous pure sarcomatous areas were noted comprising of chondrosarcoma, myxoid liposarcoma, leiomyosarcoma and rhabdomyosarcoma (inset, Fig. 1). Also, large areas of coagulative necrosis and hemorrhage were noted. The adjacent isthmus was involved by the tumor, however, the rest of the uterus with bilateral adnexae were free of tumor. The separately sent tissue from the posterior peritoneum showed a tumor with similar morphology as described above, however the omentum was free of tumor. On immunohistochemical staining, the low 
Table 1. Clinico-pathologic features of mullerian adenosarcoma with sarcomatous overgrowth cases reported in English literature

\begin{tabular}{llll}
\hline & \multicolumn{1}{c}{ Park et al. $^{5}$} & \multicolumn{1}{c}{ Comunoglu et al. $^{4}$} & \multicolumn{1}{c}{ Present case } \\
\hline Age & $37 \mathrm{yr}$ & $60 \mathrm{yr}$ & $15 \mathrm{yr}$ \\
Gross appearance & $\begin{array}{l}\text { Normal sized uterus } 2 \mathrm{~cm} \\
\text { endocervical polypoidal mass }\end{array}$ & $\begin{array}{c}\text { Normal sized uterus } 12 \mathrm{~cm} \\
\text { endocervical polypoidal mass }\end{array}$ & $\begin{array}{c}\text { Enlarged uterus with friable } \\
\text { mass replacing entire cervix }\end{array}$ \\
& $20 \mathrm{MF} / 10 \mathrm{HPF}$ & $10 \mathrm{MF} / 10 \mathrm{HPF}$ & $24 \mathrm{MF} / 10 \mathrm{HPF}$ \\
Pure sarcoma mitotic rate (MF/10 HPF) & - & $40 \%$ & $70 \%$ \\
Pure sarcoma (\%) & Absent & Absent & Present \\
Heterologous elements & No & No & Yes \\
Adjuvant chemotherapy/radiotherapy & Absent & Absent & Present and patient died of \\
Recurrence & & & disease \\
\hline
\end{tabular}

MF: mitotic figure, HPF: high power field.

grade sarcomatous areas were vimentin and CD-10 positive, thus resembling an endometrial stromal sarcoma. However, the high grade sarcomatous areas were strongly positive for vimentin and desmin. The liposarcomatous component was highlighted by S-100 immunostaining. Tumor cells were negative for estrogen and progesterone receptors. In view of the above mentioned histopathological features, a diagnosis of mullerian adenosarcoma (heterologous) with sarcomatous overgrowth of the cervix was given. The patient was followed up in the radiotherapy clinic and was given six cycles of chemotherapy followed by radiotherapy. After one year of follow up, the patient was free of tumor. However, in her last follow up 3 months previously, she presented with ascites and the CT scan revealed a recurrent tumor. The patient was lost to follow up for 1 month and on enquiring the parents, it was informed that she died of the disease.

\section{DISCUSSION}

MA belongs to the group of mixed mullerian tumors, which are infrequent uterine neoplasms, comprising of benign glandular and malignant stromal elements which may be homologous (fibroblasts and smooth muscle) or heterologous (cartilage, striated muscle, bone, etc). Although most adenosarcomas evolve in the uterine corpus/endometrium, accounting for approximately $8 \%$ of all uterine sarcomas, the neoplasm may rarely originate primarily from the uterine cervix. ${ }^{6,7}$ Only $2 \%$ of adenosarcomas arise in the cervix, with fewer than two dozen cases documented so far in the literature, however only two cases of MASO are reported in the uterine cervix and none of them had heterologous elements. ${ }^{4,5}$ Since the proportion of the glandular component to the sarcomatous component in usual adenosarcomas can vary from one area of the tumor to another, and focal areas within an otherwise typical adenosarcoma may contain rare or no glands, the diagnosis of MASO must be rendered only when a pure sarcoma, similar to, or of a higher grade than that of the underlying adenosarcoma, accounts for at least one-quarter of the tumor after thorough histological sampling of the hysterectomy specimen. ${ }^{3}$
Although MA is regarded as less aggressive, and of lower malignant potential than other malignant mullerian mixed tumors, some unfavorable prognostic factors have been listed for a MASO, high mitotic rate, heterologous elements, deep myometrial invasion, necrosis, and extrauterine spread. However, on review of the literature, the presence of myometrial invasion and sarcomatous overgrowth has been shown to be consistently associated with poor prognosis, increased risk of postoperative recurrence and fatal outcome. ${ }^{2,3}$

On review of the literature only two cases of MASO arising in the uterine cervix have been reported in middle-aged and elderly females, which presented on gross examination as polypoidal cervical masses with sizes ranging from $2 \mathrm{~cm}$ to $12 \mathrm{~cm}$. However, the present case occurred in a young unmarried girl who presented with a friable hemorrhagic tumor replacing the entire uterine cervix. ${ }^{4,5}$ On histological examination a clear demarcation was seen in the low grade and high grade sarcomatous areas with pure sarcomatous components constituting approximately $70 \%$ of the tumor, suggesting a diagnosis of MASO. The main difference between our case and the previous two reported cases is that the present case occurred in a young unmarried girl with tumor replacing the entire uterine cervix, and histologically showing malignant heterologous elements with no myometrial invasion (Table 1). Such morphological presentation of cervical MASO has not been reported previously.

To summarize, we present an extremely rare case of MASO of the uterine cervix with heterologous elements in a young girl. This case stresses that these aggressive tumors should be considered in the differential diagnosis of patients presenting with irregular vaginal bleeding and pelvic masses. Since uterine MASO is associated with poor prognosis, a thorough surgical evaluation is recommended in all such cases and with the accumulation of individual cases, such as ours, and prospective studies further experience may be gained for determining treatment options of cervical MASO.

\section{CONFLICT OF INTEREST}

No potential conflict of interest relevant to this article was reported. 


\section{REFERENCES}

1. Clement PB, Scully RE. Mullerian adenosarcoma of the uterus: a clinicopathologic analysis of ten cases of a distinctive type of mullerian mixed tumor. Cancer 1974; 34: 1138-49.

2. Clement PB, Scully RE. Mullerian adenosarcoma of the uterus: a clinicopathologic analysis of 100 cases with a review of the literature. Hum Pathol 1990; 21: 363-81.

3. Clement PB. Mullerian adenosarcomas of the uterus with sarcomatous overgrowth: a clinicopathological analysis of 10 cases. Am J Surg Pathol 1989; 13: 28-38.

4. Comunoglu N, Comunoglu C, Bassullu N, Somunkiran A, Calay Z. Mullerian adenosarcoma with sarcomatous overgrowth of the cervix: unusual large polypoid mass. Ups J Med Sci 2007; 112: 67-72.

5. Park HM, Park MH, Kim YJ, Chun SH, Ahn JJ, Kim CI, et al. Mullerian adenosarcoma with sarcomatous overgrowth of the cervix presenting as cervical polyp: a case report and review of the literature. Int J Gynecol Cancer 2004; 14: 1024-9.

6. Manoharan M, Azmi MA, Soosay G, Mould T, Weekes AR. Mullerian adenosarcoma of uterine cervix: report of three cases and review of literature. Gynecol Oncol 2007; 105: 256-60.

7. Mumba E, Ali H, Turton D, Cooper K, Grayson W. Human papillomaviruses do not play an aetiological role in Mullerian adenosarcomas of the uterine cervix. J Clin Pathol 2008; 61: 1041-4. 